\title{
Systemic lupus erythematosus serum IgG increases CREM binding to the IL-2 promoter and suppresses IL-2 production through CaMKIV
}

\author{
Yuang-Taung Juang, ${ }^{1,2}$ Ying Wang, ${ }^{1}$ Elena E. Solomou, ${ }^{1}$ Yansong Li,1 Christian Mawrin, ${ }^{1}$ \\ Klaus Tenbrock, ${ }^{1}$ Vasileios C. Kyttaris, ${ }^{1,2,3}$ and George C. Tsokos ${ }^{1,2}$ \\ ${ }^{1}$ Department of Cellular Injury, Walter Reed Army Institute of Research, Silver Spring, Maryland, USA. ${ }^{2}$ Department of Medicine, \\ Uniformed Services University of the Health Sciences, Bethesda, Maryland, USA. ${ }^{3}$ Department of Medicine, \\ Section of Rheumatology, Washington Hospital Center, Washington, D.C., USA.
}

\begin{abstract}
Systemic lupus erythematosus (SLE) T cells express high levels of cAMP response element modulator (CREM) that binds to the $I L-2$ promoter and represses the transcription of the $I L-2$ gene. This study was designed to identify pathways that lead to increased binding of CREM to the $I L-2$ promoter in SLE T cells. $\mathrm{Ca}^{2+} / \mathrm{calmodu}^{-}$ lin-dependent kinase IV (CaMKIV) was found to be increased in the nucleus of SLE T cells and to be involved in the overexpression of CREM and its binding to the $I L-2$ promoter. Treatment of normal $T$ cells with SLE serum resulted in increased expression of CREM protein, increased binding of CREM to the $I L-2$ promoter, and decreased $I L-2$ promoter activity and IL-2 production. This process was abolished when a dominant inactive form of CaMKIV was expressed in normal T cells. The effect of SLE serum resided within the IgG fraction and was specifically attributed to anti-TCR/CD3 autoantibodies. This study identifies CaMKIV as being responsible for the increased expression of CREM and the decreased production of IL-2 in SLE T cells and demonstrates that anti-TCR/CD3 antibodies present in SLE sera can account for the increased expression of CREM and the suppression of IL-2 production.
\end{abstract}

\section{Introduction}

$\mathrm{T}$ cells from humans (1) and mice (2) with systemic lupus erythematosus (SLE) produce less IL-2. Decreased IL-2 production contributes to an increased rate of infections (3) and decreased ability to generate proper activation-induced cell death, allowing $\mathrm{B}$ cell-helping subsets to survive longer (4). While exploring the reasons for the decreased production of IL-2, we found that the cAMP response element modulator (CREM) was present at increased levels in the nucleus of T lymphocytes from patients with SLE and that it bound to the -180 site of the $I L-2$ promoter. This binding has been shown to lead to transcriptional repression of $I L-2$ promoter activity $(5,6)$. However, the molecular mechanisms underlying the upregulated activity of CREM in SLE T cells remain unknown.

CREM is a transcription factor of the leucine-zipper family that also includes CREB, CREB-2, and ATF-1, -2, and -3 (7). All members share highly homologous structure in both DNA-binding and kinase-inducible domains. They differ, though, from each other in the number of activation domains they contain. CREM $\alpha$ and CREM $\beta$, unlike CREB, do not encode the Q1 and Q2 activation domains, and, therefore, they function as transcriptional suppressors (8). CREM binds to cAMP response element (CRE) either as a

Nonstandard abbreviations used: -180 /protein complex, -180 site-defined oligonucleotide/protein complex; CaMK, $\mathrm{Ca}^{2+} /$ calmodulin-dependent kinase; CaMKIV(i), inactive CaMKIV; CRE, cAMP response element; CREM, CRE modulator; EMSA, electrophoresis migration shift assay; hnRNP, heterogeneous ribonucleoprotein; SLE, systemic lupus erythematosus.

Conflict of interest: The authors have declared that no conflict of interest exists.

Citation for this article: J. Clin. Invest. 115:996-1005 (2005)

doi:10.1172/JCI200522854 homodimer or as a CREM/CREB heterodimer. Binding of CREM to the $I L-2$ promoter in the anergic T cell line A.E7 (9) and SLE $\mathrm{T}$ cells $(5,6)$ limits the production of IL- 2 .

CREM and CREB are downstream transcription factors to multiple signaling pathways triggered by diverse stimuli including stress and hormones (7). Kinases modulate the activity of CREM and CREB by altering their transcriptional and translational regulation, as well as by implementing posttranslational modifications. Kinases known to regulate the activity of CREB and CREM include PKA, PKC, ERK1, and $\mathrm{Ca}^{2+} /$ calmodulin-dependent kinase II (CaMKII) and CaMKIV $(10,11)$. The facts that PKA, ERK1, and PKC expression and/or activity have been reported to be decreased in SLE T cells (10-12) and that SLE T cells display increased TCR-mediated free intracytoplasmic $\mathrm{Ca}^{2+}$ responses (13) have provided a rationale for the exploration of the role of CaMKs in the increased expression of CREM in SLE T cells. In addition, CREM can be activated by sera through the p70 S6 kinase (14), and CaMKIV has been reported to be activated by serum in murine fetal thymic organ cultures (15). SLE sera contain various components that are not present in significant levels in normal sera, such as autoantibodies, immune complexes, and various cytokines including IFN- $\alpha$ and IL- $6(16,17)$, all of which can affect cell function. In this study we demonstrate that SLE serum IgG activates CaMKIV, which moves to the nucleus and causes increased binding of CREM to the IL-2 promoter and represses its activity.

\section{Results}

Increased expression and binding of CREM to the -180 site of the IL-2 promoter in SLE T cells. We have previously shown that SLE T cells 

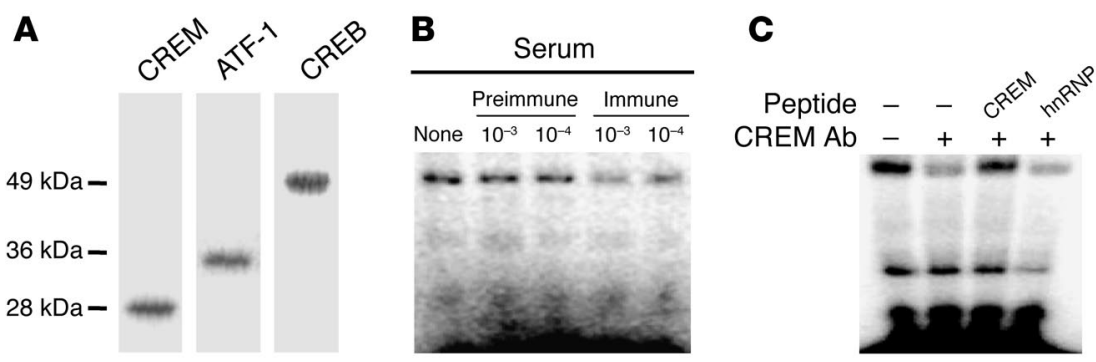

\section{Figure 1}

Characterization of a new anti-human CREM-specific antibody. (A) The new anti-CREM antibody recognizes CREM but not CREB and ATF-1. Nuclear proteins from primary $T$ cells were resolved in SDS gel and then subjected to Western blotting with the new CREM antibody. The membrane was then stripped and blotted sequentially with anti-ATF-1 and anti-CREB antibody. The anti-CREM antibody recognizes only 1 band at the site expected for CREM $\alpha$. Similarly, the anti-ATF-1 and anti-CREB antibodies recognize bands at the expected sites. (B) The new anti-CREM antibody disrupts the formation of -180 site-defined oligonucleotide/protein complex. Nuclear proteins from Jurkat T cells were incubated with the -180 oligonucleotide and $1 \mu \mathrm{l}$ of serially diluted preimmune or immune serum. (C) Human CREM-defined peptide (amino acids 21-34), but not hnRNP-defined peptide (amino acids 263-275), blocks the ability of anti-CREM antibody to disrupt the formation of -180 site-defined oligonucleotide/protein complex. Anti-CREM serum was used at $10^{-3}$ dilution in the presence or absence of $1 \mu \mathrm{M}$ of the indicated peptide.

express higher levels of both CREM mRNA (6) and protein (5) than normal $\mathrm{T}$ cells do. Furthermore, we have demonstrated that increased CREM binding to the -180 site of the IL- 2 promoter leads to a decrease in promoter activity and IL-2 production $(5,6)$. To further confirm the above findings, we produced a new specific anti-CREM antibody that does not cross-react with CREB or ATF-1 (Figure 1A). This antibody efficiently disrupted the formation of -180 site-defined oligonucleotide/protein complex (Figure 1B) in shift assays. The presence of the immunizing peptide, but not of a control peptide, inhibited the ability of the anti-CREM antibody to disrupt the $-180 /$ protein complex (Figure 1C). In agreement with previously reported data (5), SLE T cells displayed increased binding to the -180 site of the IL- 2 promoter in shift assays (Figure 2A; $n=11, P<0.05$ ). Using the new specific antibody in super-shift assays (Figure $2 \mathrm{~B}$ ), we determined that the majority of the complex in SLE T cells represented CREM rather than CREB binding. In the experiment presented in Figure 2B, the new anti-CREM antibody decreased the intensity of the complex by $85 \%$, whereas an anti-CREB antibody decreased it by $40 \%$. The presence of both antiCREM and anti-CREB antibodies simultaneously in the shift reaction eliminated the majority of the binding (data not shown). The complex was also disrupted in the presence of wild-type but not in the presence of a mutated cold oligonucleotide (Figure 2C).

SLE sera stimulate the expression of CREM and its binding to the -180 site of the IL-2 promoter in normal T cells. Because SLE sera have been reported to alter immune cell function $(16,17)$ and because the activity of CREB and CREM is regulated by a number of signaling pathways initiated by extracellular stimuli, we considered whether the dysregulated activity of CREM in SLE T cells results from a factor(s) present in SLE sera. Therefore, we asked whether SLE sera can

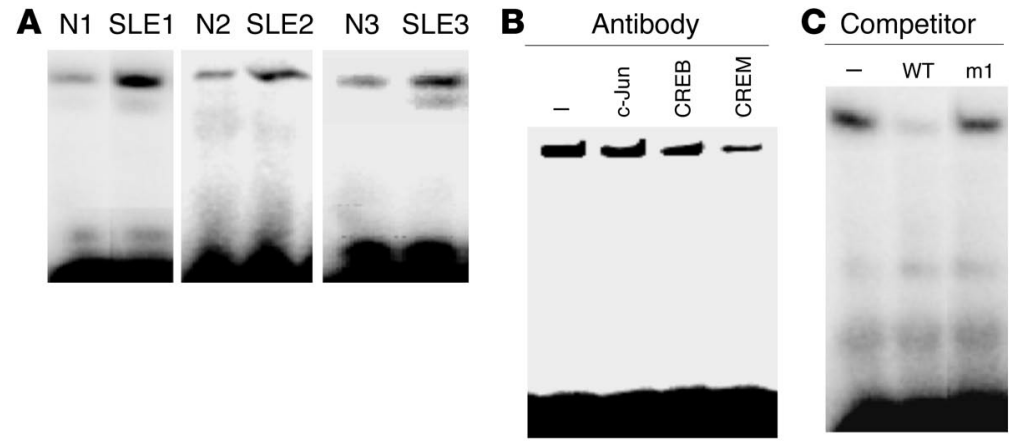

Figure 2

Increased binding of CREM to the -180 site of the IL-2 promoter in SLE T cells. (A) Nuclear proteins were incubated with a ${ }^{32} \mathrm{P}$-labeled oligonucleotide encoding the -180 site of the IL-2 promoter as described in Methods. Shown are 3 independently conducted EMSAs, each containing 1 pair of simultaneously purified normal (N) and SLE T cells. (B) Nuclear proteins from an SLE patient were incubated with the -180 oligonucleotide with or without antibody against CREB, CREM, or c-Jun for 15 minutes, followed by shift analysis. (C) Nuclear proteins from primary T cells were incubated with ${ }^{32} \mathrm{P}$-labeled oligonucleotide encoding the -180 site of the IL-2 promoter. Where indicated, unlabeled wild-type or mutant oligonucleotides containing 2 mutated nucleotides were also added into the reaction. 

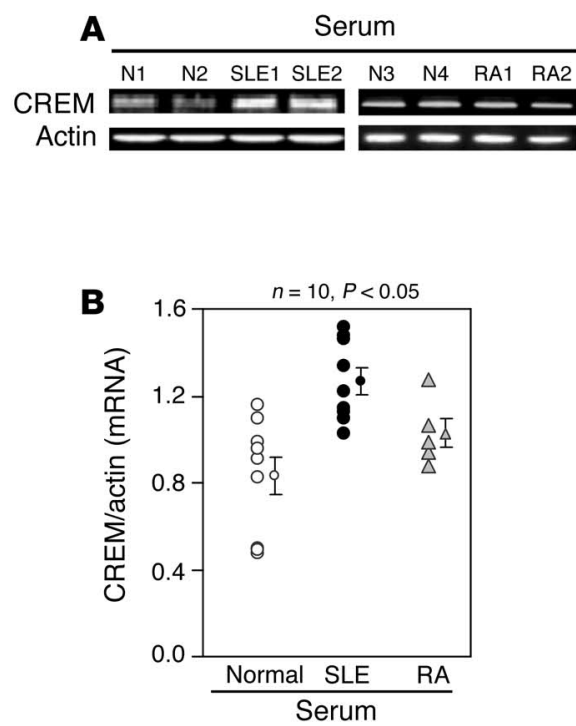
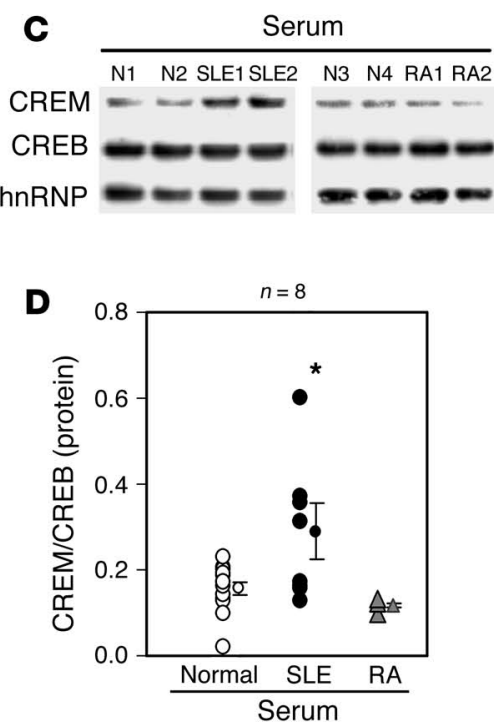
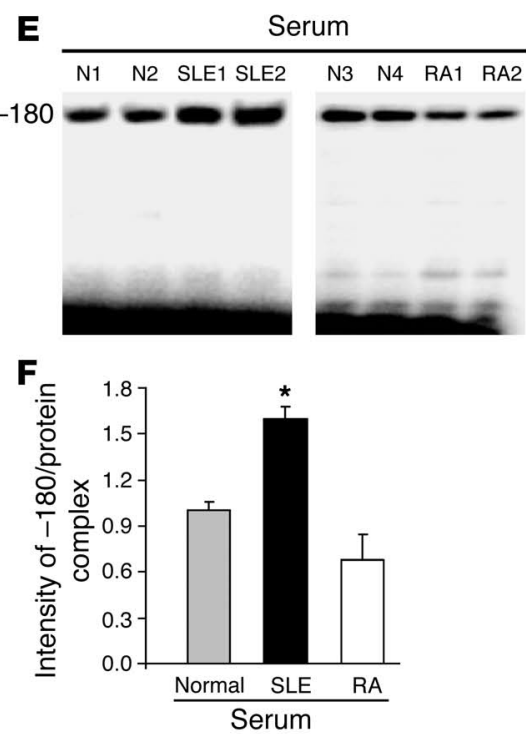

Figure 3

SLE sera stimulate the expression of CREM and its binding to the -180 element of IL-2 promoter. (A) SLE sera induce CREM mRNA. Normal T cells were stimulated with normal, RA, or SLE sera for 2 hours before RNA was analyzed for the expression level of CREM and actin. (B) Cumulative analysis of the effect of SLE sera on the expression of CREM mRNA. The $y$ axis represents the ratio between the mRNA expression levels of CREM and those of actin in normal T cells treated with SLE, RA, or normal serum. (C) SLE sera induce the expression of CREM in the nucleus. Normal T cells were cultured for 2 hours in RPMI containing 1\% serum from normal, RA, or SLE patients. Nuclear proteins were harvested and assayed using Western blotting with the indicated antibodies. Shown are 2 representative Western blots, each containing simultaneously purified nuclear proteins from normal T cells treated with normal, RA, or SLE serum. (D) Cumulative effect of SLE sera on the expression of nuclear CREM protein in normal T cells. The $y$ axis represents the ratio of the expression levels of CREM to those of CREB protein in normal T cells treated with normal, RA, or SLE sera. (E) Increased formation of -180 complex in normal T cells treated with SLE sera. Nuclear proteins from normal $T$ cells treated with sera from normal, RA, or SLE patients were analyzed by EMSA to measure their binding activity to oligonucleotides encoding the -180 element. (F) The $y$ axis represents the $-180 /$ protein complex intensity in the T cells treated with SLE, RA, or normal serum. ${ }^{*} P<0.05$.

through various signaling pathways. Among the kinases known to activate CREM, PKA, PKC, and ERK have been reported to be defective in SLE T cells (10-12), while the activity of CaMKs that are known to regulate the activity of CREM has not been studied. Furthermore, SLE T cells are known to display increased TCRmediated free intracytoplasmic $\mathrm{Ca}^{2+}$ response (13), and $\mathrm{Ca}^{2+}$ concentration is known to regulate the activity of CaMKs (18). Therefore, we investigated the role of the CaMKs in the activation of CREM. Although CaMKs are located in both the cytoplasm and the nucleus, it is the nuclear CaMKs that directly activate transcription factors. Because not all CaMKs migrate to the nucleus (19), we focused on CaMKIV and CaMKII, which can migrate and localize to the nucleus. We purified cytoplasmic and nuclear proteins from normal and SLE T cells and determined the CaMKIV levels in each compartment. As shown in Figure 4, SLE T cells expressed more CaMKIV in the nuclear compartment than normal $\mathrm{T}$ cells. No correlation was identified between disease activity and the densitometric values of CaMKIV expression. When the nuclear levels

\section{Figure 4}

Increased expression of CaMKIV in the nucleus of SLE T cells. (A) Both cytoplasmic and nuclear proteins were isolated from normal and SLE $T$ cells. Western blots were then conducted by sequential blotting of the membrane with antibody against CaMKIV or CaMKII. Representative blots from 2 patient-control pairs are shown. (B) Eleven pairs were studied. Nuclear densitometric readings for CaMKIV, but not CaMKII, are higher $(P<0.01)$ in SLE T cells than in normal T cells. ${ }^{*} P<0.05$. of CaMKIV were elevated, the cytoplasmic levels were decreased, which suggests that enhanced nuclear import is responsible for the increased levels of nuclear CaMKIV (Figure 4A). In contrast, normal $\mathrm{T}$ cells had higher levels of CaMKII than SLE T cells in the nucleus (Figure 4, A and B). Densitometric analysis of data from 11 patients with SLE and 11 normal individuals demonstrated higher levels of CaMKIV, but not CaMKII, in the nucleus of SLE T cells compared

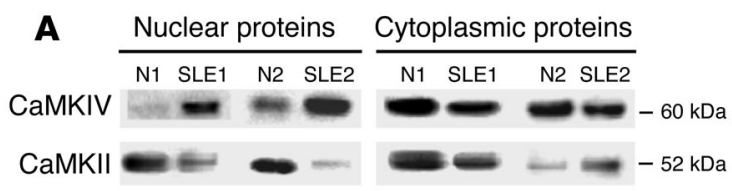

B

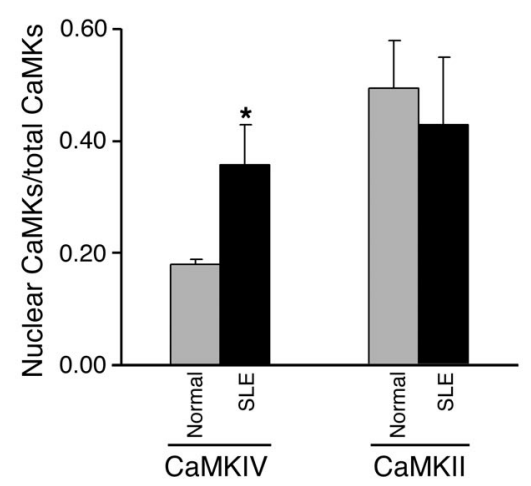




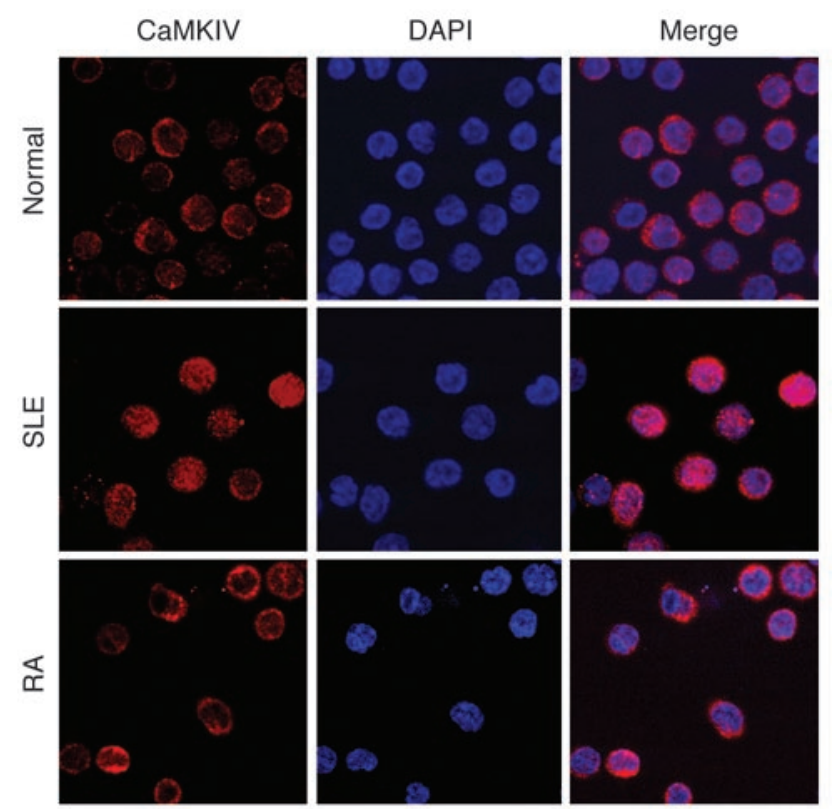

Figure 5

CaMKIV localization in normal and SLE T cells. Primary T cells from either normal or human SLE patients were stained with Cyanin 3-conjugated anti-CaMKIV or DAPI, and the images were merged as indicated. Five hundred cells were evaluated from each sample. Magnification, $\times 1000$.

with normal T cells $(P<0.05)$. Next we used confocal microscopy to visualize the localization of CaMKIV. In both normal and RA $\mathrm{T}$ cells CaMKIV was detected primarily in the cytoplasm, whereas in SLE T cells CaMKIV was detected in the nucleus of the cells and to a lesser extent in the cytoplasm. Specifically, $41.4 \% \pm 8.8 \%$ of SLE $\mathrm{T}$ cells have more CaMKIV in the nucleus than in the cytoplasm, compared with $7.3 \% \pm 3.4 \%$ of normal and $10.1 \% \pm 3.1 \%$ of RA T cells $(n=3, P<0.05$ comparing SLE with normal). Representative cell-staining data are shown in Figure 5 . These data suggest that CaMKIV but not CaMKII is activated in a manner that promotes its translocation to the nucleus of SLE T cells.

Overexpression of inactive CaMKIV blocked the effect of SLE sera on the formation of $-180 /$ protein complex. To confirm whether the enhanced expression of CREM and its subsequent binding to the -180 site of the IL-2 promoter are mediated through activation of CaMKIV, normal $\mathrm{T}$ cells were transiently transfected with either empty vector or a construct expressing inactive CaMKIV [CaMKIV(i)] and then were treated with SLE sera. As shown in Figure 6A, forced expression of CaMKIV(i) diminished significantly the formation of the SLE serum-induced $-180 /$ protein complex. In contrast,

\section{Figure 6}

Overexpression of CaMKIV(i), but not overexpression of CaMKII(i), abolishes the SLE serum-induced -180 site/protein complex formation. (A) Normal T cells were transfected with control plasmid or plasmids encoding catalytically inactive CaMKII or CaMKIV [CaMKII(i) or CaMKIV(i)]. Three hours later, cells were treated with SLE, RA, or normal sera for 2 more hours and then harvested for EMSA analysis. (B) Cumulative data from 5 experiments are presented. (C) CaMKII(i) does not abolish the effect of SLE serum on the formation of the $-180 /$ protein complex. (D) Cumulative data are presented. ${ }^{*} P<0.05$.
CaMKIV(i) did not alter the effect of normal and RA sera. In Figure $6 \mathrm{~B}$, cumulative data are shown from 5 experiments in which the effect of SLE, normal, or RA sera on the formation of -180/ protein complex was studied in cells transfected with CaMKIV(i) or pcDNA. The effect of SLE serum was limited significantly $(P<0.05)$ in the presence of CaMKIV(i), whereas the effect of normal and RA serum was not. To demonstrate that sera exerted their effect only through CaMKIV, we repeated these experiments using an inactive form of CaMKII. As can be seen in Figure 6, C and D, CaMKII(i), unlike CaMKIV(i), did not alter the effect of SLE sera on the formation of $-180 /$ protein complex. These data indicate that the SLE serum-induced increase in the formation of -180 / CREM protein complex is dependent on the presence of CaMKIV.

CaMKIV enhanced the expression of CREM and its binding to the -180 site of the IL-2 promoter in SLE T cells. Next we compared the effect of overexpression of CaMKIV on the formation of -180 /protein complex in normal and SLE T cells. We established that the available CaMKIV vector, when transferred into $\mathrm{T}$ cells, resulted in CaMKIV protein expression. As shown in Figure 7A, transfection of normal $\mathrm{T}$ cells with a CaMKIV expression construct resulted in a time-dependent increase in the expression of CaMKIV protein. Next, SLE T cells were transiently transfected with plasmids overexpressing wild-type CaMKIV and then were treated with PMA and ionomycin to activate CaMKIV. Transfection of normal T cells with CaMKIV expression or empty vectors did not affect the ability of cells to mobilize $\mathrm{Ca}^{2+}$ in response to ionomycin 3 hours later (data not shown). As shown in Figure 7B, overexpression of CaMKIV stimulated the expression of CREM but not heterogeneous ribonucleoprotein (hnRNP) in SLE T cells. The increased expression of CREM that was observed in SLE T cells following the expression of CaMKIV was associated with the increased binding of protein to the -180 site-defined oligonucleotide (Figure 7C).

We then asked whether overexpression of CaMKIV alone was sufficient to induce the $-180 /$ protein complex in normal $\mathrm{T}$ cells. Normal T cells were transfected with an empty vector or a plasmid expressing wild-type CaMKIV and then were treated with PMA and ionomycin. As shown in Figure 7D, in contrast to SLE T cells, where CaMKIV significantly enhanced the formation of the -180 / protein complex, there was no consistent effect in normal $\mathrm{T}$ cells in which CaMKIV was overexpressed. Time-curve analysis of normal

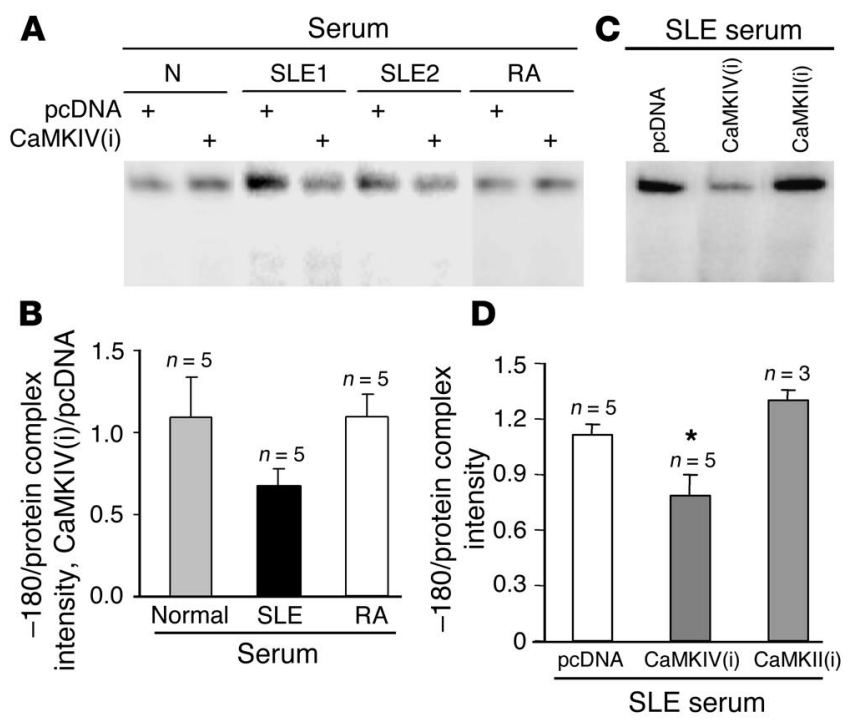



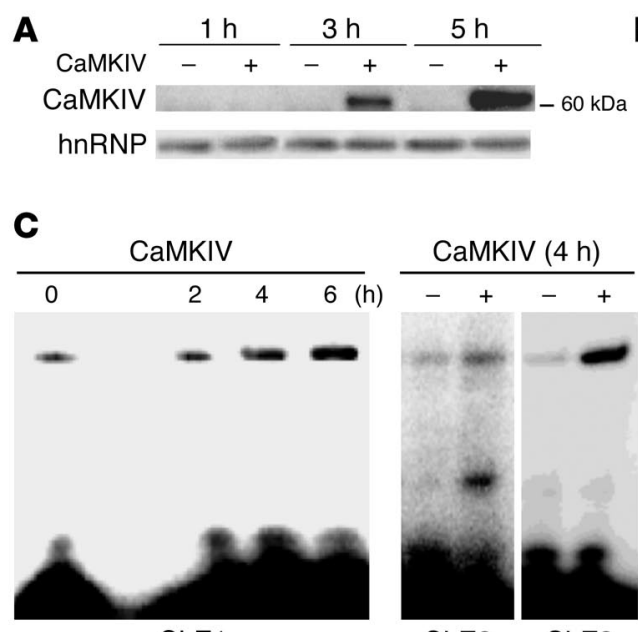

SLE1

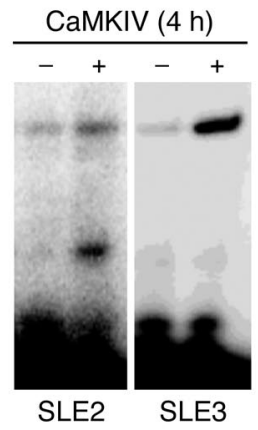

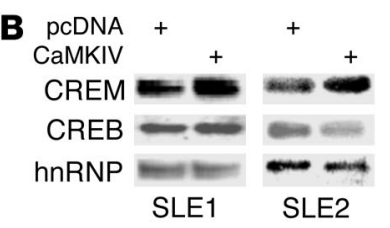

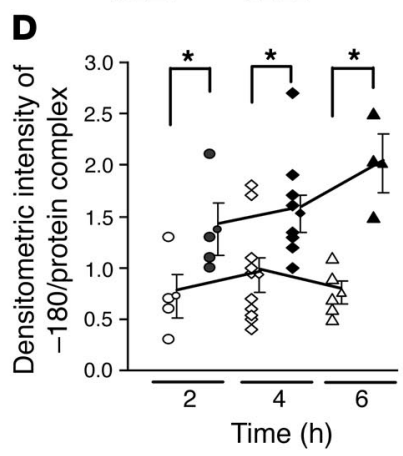

Figure 7

CaMKIV upregulates the expression of CREM and its binding to the -180 site of the IL-2 promoter in SLE T cells. (A) Normal T cells were transfected with a CaMKIV expression construct for the indicated time, and the lysates were blotted with an anti-CaMKIV or an anti-hnRNP (control) antibody. (B) SLE T cells were transfected with control plasmid or a plasmid overexpressing CaMKIV and then were treated with PMA and ionomycin. Four hours later, nuclear proteins were purified, and Western blotting was conducted by sequential use of antibodies as indicated. An antibody against hnRNP was used as control. (C) SLE T cells were transfected with control plasmid or a plasmid expressing wild-type CaMKIV and then were treated with PMA and ionomycin. At the indicated time points, cells were harvested, and EMSA was conducted using the oligonucleotide encoding the -180 site of the $I L-2$ promoter. (D) Cumulative time-curve results of the effect of CaMKIV overexpression in normal and SLE T cells. The $y$ axis represents the ratio of the intensity of the -180 /protein complex in the CaMKIV-transfected cells to that in the cells transfected with control plasmid. Normal and SLE T cells were subjected to the experimental protocol detailed in C. Filled symbols, SLE T cells; open symbols, normal T cells. ${ }^{\star} P<0.05$.

and SLE cells demonstrated that CaMKIV increased the intensity of the $-180 /$ protein complex formation only in SLE T cells. This effect was prominent at the 6-hour time point. This finding indicates that overexpression of CaMKIV in normal $\mathrm{T}$ cells is not sufficient to reproduce the effect of SLE sera.

CaMKIV suppresses the -180 element-driven luciferase activity in SLE but not in normal $T$ cells. We have reported that increased amounts of $-180 / C R E M$ protein complex result in suppression of $I L-2$ promoter activity in SLE T cells (5). To test whether CaMKIV-mediated increased binding of CREM to the IL-2 promoter is associated with decreased $I L-2$ promoter activity, we compared the effect of CaMKIV on the -180 site-driven luciferase activity in normal and SLE T cells. The activity of this construct reflects that of the proximal IL-2 promoter and IL-2 production (5). A luciferase reporter construct driven by 2 copies of the -180 site on the IL-2 promoter was cotransfected into normal $(n=5)$ and SLE $(n=4) \mathrm{T}$ cells with

\section{Figure 8}

CaMKIV suppresses the -180 site-driven reporter activity in SLE T cells. Freshly purified normal and SLE T cells were transiently cotransfected with the luciferase reporter construct driven by the -180 site of the IL-2 promoter (2 copies placed in tandem) and with control plasmid or a plasmid expressing wild-type CaMKIV, and then stimulated with PMA and ionomycin. The $y$ axis represents the $\beta$-gal-normalized luciferase activity, which was assayed 6 hours after transfection. ${ }^{\star} P<0.05$. either control plasmid or CaMKIV expression vector. In accordance with previously published data (5), normal $\mathrm{T}$ cells had 3.5 times higher basal -180 site-driven reporter activity than SLE T cells, and this difference inversely correlated with the respective basal CREM-binding activity. The difference was not due to the differential transfection efficiency of normal and SLE T cells, since the activity of the cotransfected galactosidase was comparable in normal and SLE T cells. We arbitrarily set the basal activity level of the - 180 site-driven luciferase construct at 100 in normal and SLE T cells. As shown in Figure 8, the -180 reporter activity was not affected significantly by CaMKIV in normal $\mathrm{T}$ cells, while it was significantly downregulated $(P<0.05)$ in SLE T cells.

Priming of normal $T$ cells with SLE serum before transfection with CaMKIV enhances the formation of $-180 /$ protein complex. We next determined whether incubation of normal CaMKIV-transfected T cells with SLE sera could facilitate the formation of $-180 /$ protein complex. To this end, we treated normal $\mathrm{T}$ cells with SLE sera for 2 hours, transfected them with a CaMKIV expression construct, and analyzed them 3 hours later. As shown in Figure 9, A (representative) and B (cumulative), SLE sera significantly augmented $(n=4$, $P<0.05)$ the formation of the $-180 /$ protein complex in normal $\mathrm{T}$ cells overexpressing CaMKIV. These data suggest that SLE sera are required for the optimization of the effect of CaMKIV on the formation of the $-180 /$ protein complex.

Priming of CaMKIV-transfected normal T cells with SLE sera suppresses proximal IL-2 promoter activity and IL-2 production. To establish that priming of normal cells with SLE sera suppresses the transcriptional activity of the IL-2 promoter, we incubated normal T cells with SLE sera and transfected them with CaMKIV and a proximal IL-2 promoter reporter construct. As is shown in Figure 10A (cumulative data from 4 experiments), exposure of normal T cells to SLE sera suppressed the activity of the $I L-2$ promoter by approximately $50 \%$ $(P<0.03)$. The effect on the promoter activity was reflected in decreased IL-2 production by these cells. As shown in Figure $10 \mathrm{~B}$, pretreatment of normal cells with SLE serum resulted in significantly decreased IL-2 production $(n=5, P<0.03)$. Therefore, exposure of normal T cells overexpressing CaMKIV (through

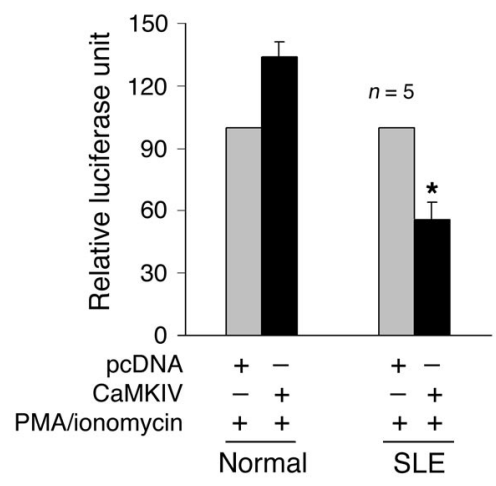


A

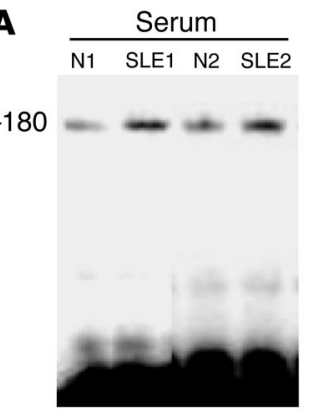

Figure 9

SLE sera enhance CaMKIV-mediated activation of the -180 /protein complex in normal T cells. (A) SLE sera augment the effect of CaMKIV on the formation of the $-180 /$ protein complex in normal T cells. Normal T cells were primed with normal or SLE sera for 2 hours; cells were then transfected with CaMKIV expression plasmids and treated with PMA and ionomycin. Three hours later, cells were harvested, and EMSA was performed by incubation of nuclear proteins with the $-180 /$ protein complex. (B) Cumulative results from 4 experiments $(P<0.05)$.

transfection) to SLE sera results in transcriptional repression of the IL-2 gene and decreased production of IL-2.

SLE serum IgG is responsible for the increased -180 site of the IL-2 promoter/protein complex formation and the decreased production of IL-2 by normal $T$ cells. In order to identify the component(s) of SLE sera responsible for the increased formation of -180 /protein complex, we fractionated SLE sera into IgG and non-IgG fractions (Figure 11A). As shown in Figure 11B (one representative experiment) and Figure 11C (cumulative data, $n=4$ ), the effect of the SLE sera on the formation of -180 /protein complex resided primarily within the IgG fraction. Similarly, the IgG fraction was found to be responsible for the decreased production of IL-2 by normal $\mathrm{T}$ cells.

SLE sera have been extensively shown to contain anti-lymphocytic antibodies directed against various determinants, including antibodies against CD $45, C D 4$, and TCR $\alpha \beta$ chain (17). To address directly whether antibodies directed against these determinants can reproduce the effects observed with SLE Ig, we treated normal cells with antibodies against CD3 (surrogate for TCR $\alpha \beta$ ), CD4, CD28, and CD45. As shown in Figure 12A, only anti-CD3 antibody activated CaMKIV. $T$ cells treated with anti-CD3 antibody expressed significantly more CaMKIV $(P<0.01, n=4)$ in nuclear extracts compared with $\mathrm{T}$ cells stimulated with control antibody (Figure 12B), and this was verified by confocal microscopy experiments, which demonstrated that CaMKIV indeed translocated to the nucleus of the cells (Figure 12C). None of the other antibodies had a significant effect on the expression of CaMKIV. Besides increasing the expression of CaMKIV in the nucleus of $\mathrm{T}$ cells, anti-CD3 increased the expression of CREM (Figure 12D) and the formation of -180 /protein complex (Figure 12E).

The TCR/CD3 complex represents the target for SLE serum IgG that is responsible for the increased expression of-180/protein complex formation. Having established that anti-CD3 antibodies can cause translocation of CaMKIV from the cytoplasm to the nucleus and increase the expression of CREM and its binding to the -180 site of the IL-2 promoter, we considered that the TCR/CD3 complex may represent the ligand for the SLE IgG that is responsible for the observed events. To this end, we first treated Jurkat T cells or J.EMS.T3.3 cells (a Jurkat $\mathrm{T}$ cell subline missing the TCR/CD3 complex) with $1 \%$ normal or SLE sera for 3 hours and subjected nuclear extracts to a shift assay using the -180 oligonucleotide. As shown in Figure 13A (representative) and Figure 13B (cumulative, $n=4$ ), the intensity of the $-180 /$ protein complex increased significantly $(P<0.05)$ only in the TCR/CD3-positive cells and not in TCR/CD3-negative cells treated with SLE sera. The intensity of the -180 /protein complex was comparable in TCR/CD3-positive and -negative cells treated with normal sera. Second, we adsorbed SLE sera on TCR/CD3-positive and-negative Jurkat $\mathrm{T}$ cells and then used them to treat normal $\mathrm{T}$ cells. Figures 13C and 12D (cumulative data using 4 different SLE sera) demonstrate that adsorption of SLE sera on TCR/CD3-positive cells only and not on TCR/CD3-negative T cells decreased significantly $(P<0.05)$ their ability to enhance the expression of the $-180 /$ protein complex in normal T cells. Finally, we isolated the IgG fraction from SLE sera adsorbed on TCR/CD3-positive or-negative $T$ cells and used it to treat normal T cells (Figure 13E; 1 of 2 similar experiments is shown). Again, the IgG fraction from sera adsorbed on TCR/CD3-positive cells lost its ability to enhance the formation of -180 /protein complex in normal T cells, whereas the IgG fraction from sera adsorbed on TCR/CD3-negative Jurkat $\mathrm{T}$ cells did not.

\section{Discussion}

We previously demonstrated that CREM is overexpressed in SLE $\mathrm{T}$ cells, binds to the IL-2 promoter, and limits its activity, resulting in decreased production of IL-2 $(5,6)$. In this report we show that SLE sera cause normal $T$ cells to acquire the molecular phenotype of SLE T cells, that is, increased CREM expression with decreased IL-2 promoter activity and IL-2 expression.

SLE sera can induce the expression of CREM in normal $\mathrm{T}$ cells at both the protein and the mRNA level. The effect on CREM mRNA suggests that SLE sera cause either enhanced CREM gene transcription or increased CREM mRNA stability. The effect of SLE sera was limited to CREM, since the expression levels of CREB were not affected. This finding is consistent with previously published data that indicated that the expression of CREM can be induced independently of that of CREB $(20,21)$. It is possible that SLE sera, either through the demonstrated effect on CaMKIV or through other signaling pathways, may affect CREM mRNA stability.

CREM is the effector molecule of multiple signaling pathways that involve kinases such as PKA, PKC, p70 S6 kinase (22), and CaMKs
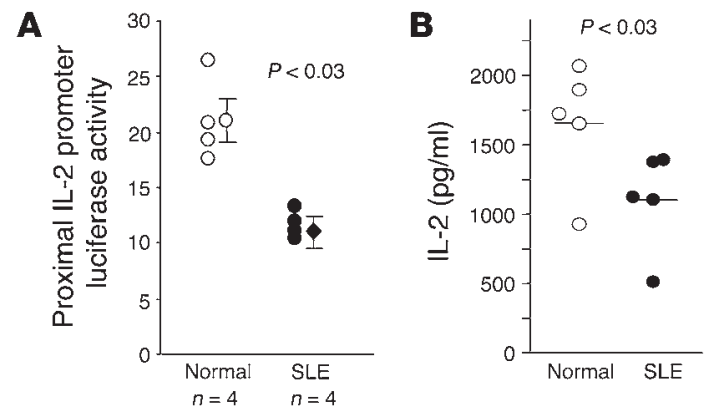

\section{Figure 10}

SLE sera suppress IL-2 production by normal T cells. (A) Normal T cells were incubated for 16 hours with $1 \%$ SLE serum and then cotransfected with a CaMKIV expression vector and a proximal $(-575$ to +57 ) IL-2 promoter reporter construct. Three hours later, cells were treated with PMA and ionomycin, and after 5 hours the luciferase activity was measured and normalized against $\beta$-gal activity to control for transfection efficiency. (B) Cells were subjected to the same treatment, and the produced IL-2 was determined using an ELISA. 


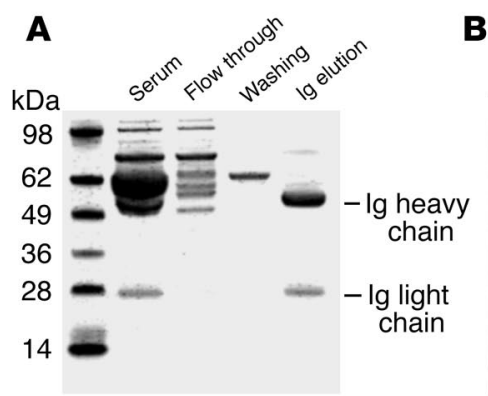

B $\frac{\text { Serum }}{N \quad \text { SLE }} \frac{\text { through }}{N \text { SLE }} \frac{\text { elution }}{N \text { SLE }}$

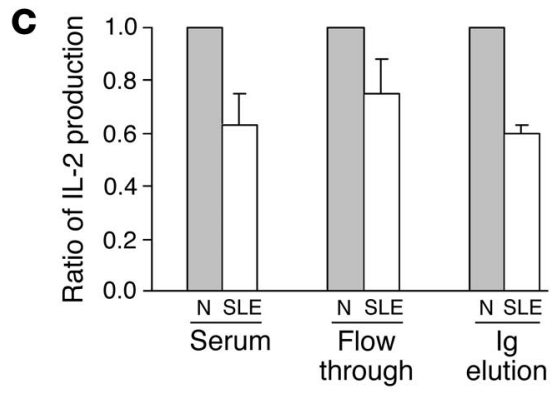

(21). The fact that both PKA and PKC as well as ERK are defective in SLE T cells (10-12) has directed our attention to the study of the role of CaMKs in the overexpression of CREM in SLE T cells. This rationale was further supported by the facts that SLE T cells display enhanced $\mathrm{Ca}^{2+}$ responses (13) and that $\mathrm{Ca}^{2+}$ is typically required for the function of CaMKs (18). More importantly, we found enhanced expression of CaMKIV in the nucleus of SLE T cells. This finding is of pathophysiological importance for 2 reasons. First, CaMKIV has a selective immune cell expression pattern, as it is expressed primarily by $T$ cells whereas $B$ cells and macrophages have no detectable

\section{Figure 11}

SLE serum IgG promotes the binding of protein to the -180 site of the IL-2 promoter and decreases the production of IL-2 in normal T cells. (A) SLE and normal sera were fractionated (see Methods) and subjected to electrophoresis. (B) Exposure of normal T cells to SLE sera and $\mathrm{IgG}$ fractions $(2 \mu \mathrm{g} / \mathrm{ml})$ from SLE sera increases the formation of $-180 /$ protein complex. Data from 1 experiment $(n=4)$ are shown. (C) SLE sera and IgG fractions of SLE sera $(n=4)$ limit the ability of normal T cells to produce IL-2. IL-2 production in the presence of normal sample was set at 1 (gray bars).

CaMKIV (23-25). Second, as shown in Figures 4 and 5, CaMKIV is located in the cytoplasmic compartment of normal T cells, whereas in SLE T cells CaMKIV resides primarily in the nucleus. Therefore, CaMKIV is aberrantly regulated in SLE T cells and may contribute to dysregulation of gene expression.

There is a substantial cross-talk between CaMKIV and other kinases that activate CREM. In particular, CaMKIV is negatively regulated by both PKA and PKC $(26,27)$, both of which have been reported to be less active in SLE T cells compared with normal $\mathrm{T}$ cells $(10,12)$. Also, it has been shown that CaMKII negatively regulates the effects of CaMKIV on the activity of both CREB and ATF-1 (28). In Figure 4 we showed that, in SLE T cells, increased nuclear levels of CaMKIV are associated with decreased levels of CaMKII. This finding is in agreement with a previous report that CaMKIV can antagonize the effect of CaMKII by inhibiting its nuclear translocation (29). Therefore, it appears that CaMKIV exerts a dominant effect in SLE T cells that remains unopposed by PKA, PKC, and CaMKII because the activity of all 3 kinases is decreased.

After identifying that CaMKIV is responsible for the induction of the $-180 /$ protein complex in SLE T cells, we tested whether overexpression of CaMKIV is sufficient to reproduce the enhanced formation of $-180 /$ protein complex in normal $\mathrm{T}$

\section{Figure 12}

Anti-CD3 antibody activates CaMKIV and induces CREM binding to the -180 site of the IL-2 promoter in normal T cells. (A) Normal T cells were treated with either control antibody (Cont.; normal murine IgG, 1:100) or antibodies against CD3 (1:100), CD28 (1:200), CD4 $(1: 200)$, or CD45 (1:200) for the indicated time period. Nuclear and cytoplasmic proteins were blotted with antibody against CaMKIV, hnRNP, or actin. (B) Cumulative data from 4 experiments. ${ }^{* *} P<0.01$. (C) Normal T cells treated as in $\mathbf{A}$ were fixed and stained with anti-CaMKIV antibody or DAPI. Shown are the merged pictures after cells were stained with antibodies against CaMKIV and DAPI (which defines the location of the nucleus). Magnification, $\times 1000$. (D) Nuclear proteins isolated from $T$ cells treated with anti-CD3 antibody for 3 hours were blotted sequentially with antibodies against CaMKIV, CREM, and hnRNP. (E) Nuclear proteins isolated from $T$ cells treated with antiCD3 antibody for 3 hours were incubated with ${ }^{32} \mathrm{P}$-labeled -180 site oligonucleotide.

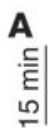
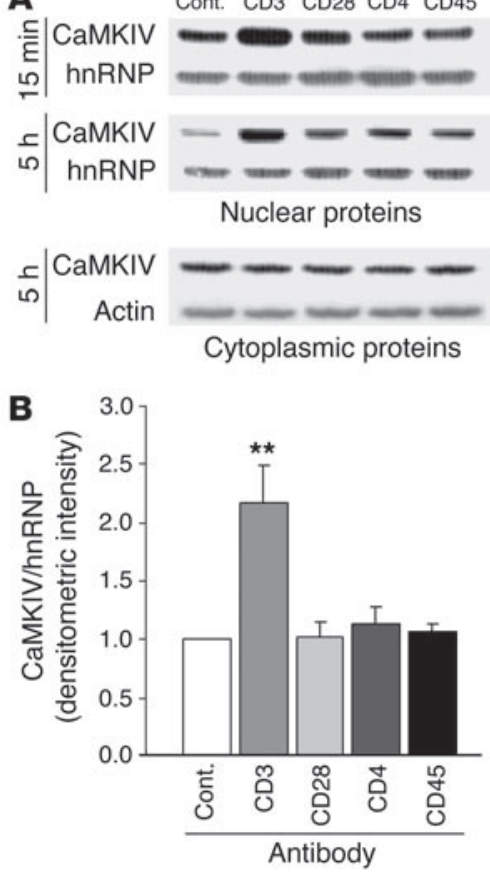

C
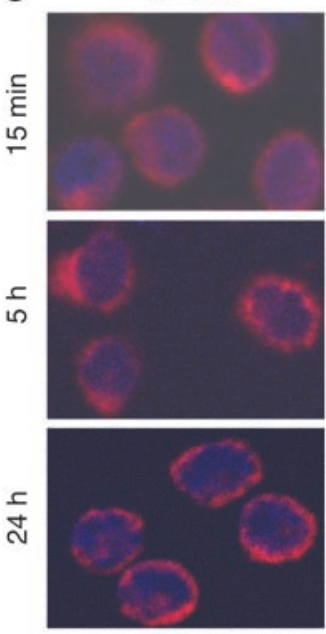

CaMKIV (red), DAPI (blue)

D

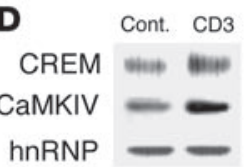

$\mathrm{CD} 3$
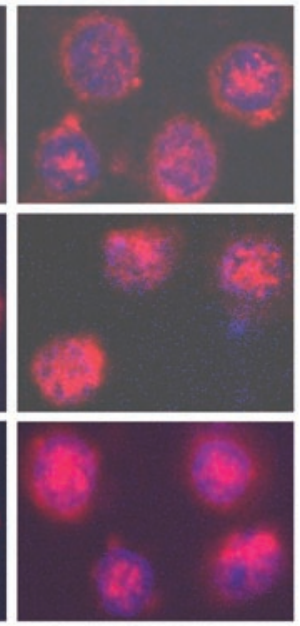

E

Cont. CD3

-180 site 
A
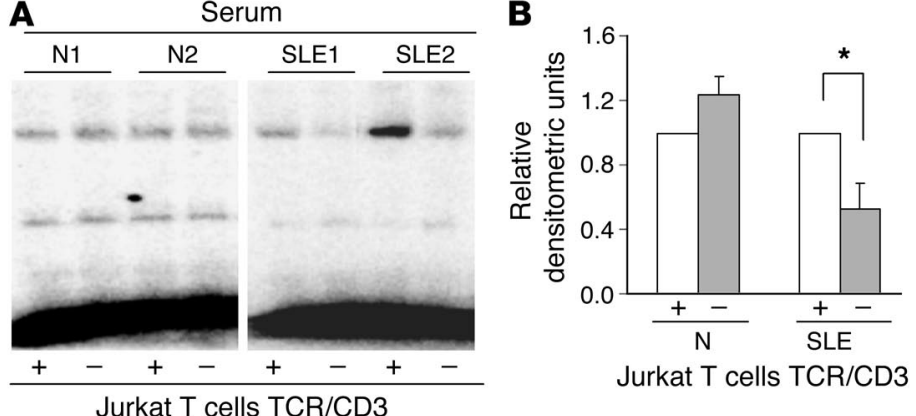

Jurkat $T$ cells TCR/CD3
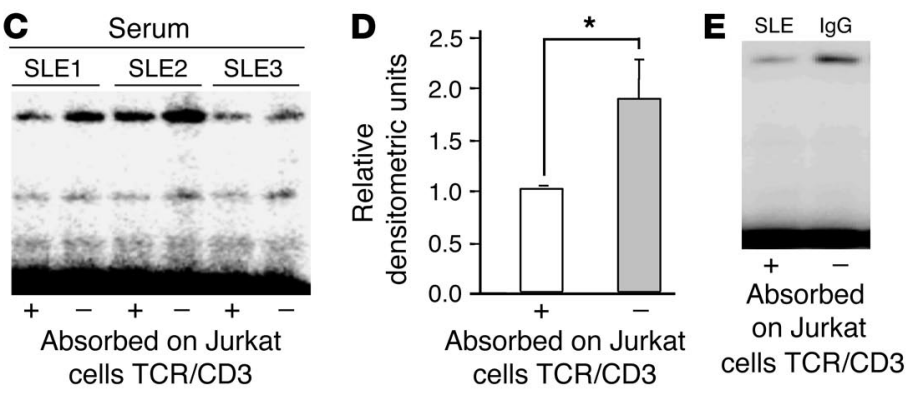

Figure 13

The TCR/CD3 complex represents the ligand for SLE serum IgG that is responsible for the increased expression of $-180 /$ protein complex formation. (A) Wild-type Jurkat T cells or J.EMS.T3.3 cells (a Jurkat T cell subline missing the TCR/CD3 complex) were treated with $1 \%$ normal or SLE sera for 3 hours, and nuclear extracts were subjected to a shift assay using the -180 oligonucleotide. (B) Cumulative data $(n=4)$ are shown. The $y$ axis indicates relative densitometric units with the effect on TCR/CD3-positive cells set at 1. ${ }^{\star} P<0.05$. (C) Normal or SLE sera were adsorbed for 30 minutes at room temperature and another 30 minutes at $4^{\circ} \mathrm{C}$ on either Jurkat or J.EMS.T3.3 $\mathrm{T}$ cells and used to treat normal T cells for 3 hours. Nuclear extracts were subjected to a shift assay using the -180 oligonucleotide. (D) Cumulative data showing the effect of adsorption of SLE sera $(n=4)$ on TCR/CD3-positive and -negative Jurkat T cells. The $y$ axis indicates relative densitometric units with the effect of sera adsorbed on TCR/CD3-positive cells set at 1 . ${ }^{*} P<0.05$. (E) SLE sera were adsorbed on TCR/CD3-positive and -negative cells, and the IgG fraction was isolated and used to treat normal $T$ cells for 3 hours. Nuclear extracts were isolated and subjected to a shift assay using the -180 oligonucleotide. One of 2 similar experiments is shown.

cells. Overexpression of CaMKIV in normal T cells did not have a consistent effect on the formation of the CREM/-180 complex. CaMKIV enzymatic activity is regulated at least at 3 levels: (a) the binding of $\mathrm{Ca}^{2+} /$ calmodulin to CaMKIV, (b) autophosphorylation of CaMKIV at threonine 196 and several other serine residues (30), and (c) the phosphorylation of CaMKIV at a number of amino acids near its $\mathrm{N}$-terminal by CaMK kinase (CaMKK) (31). The complexity of the regulation of the activity of CaMKIV suggests that in both normal and SLE T cells the level of its activity at any time point must represent the effect of multiple mechanisms, and that it may range significantly.

We considered that SLE disease activity may influence the intensity of the -180 /protein complex formation or the presence of CaMKIV in the nucleus of the cells. In the studied sample of patients, we failed to detect any association. Although the sample size may be small for statistically powerful conclusions, the lack of association with disease activity is consistent with our previous report that the increase in expression and binding of CREM to the IL-2 promoter is independent of the disease activity (5).
SLE sera contain various components that are not present in significant levels in normal sera that could, individually or acting together, cause translocation of CaMKIV to the nucleus, increased expression of CREM, and increased binding of protein to the -180 site of the IL-2 promoter. These components, such as autoantibodies (17), immune complexes, and various cytokines, including IFN- $\alpha$ (16) and IL-6 (32), have been claimed to affect cell function. In the present study we considered that SLE IgG could be responsible for the increased binding of protein to the -180 site. Indeed, the IgG fraction of SLE serum was found to be responsible for the observed effect. SLE sera contain anti-lymphocytic antibodies directed against various determinants, including TCR $\alpha \beta, C D 4$, and CD 45 (17). In this study, we demonstrate that anti-CD3 antibody, used as a surrogate for anti-TCR $\alpha \beta$ antibody, can activate CaMKIV and increase the -180 /protein complex (Figure 12). More importantly, adsorption of SLE sera on TCR-positive, but not TCR-negative, Jurkat cells limited their ability to enhance the expression of $-180 /$ protein complex in normal T cells; in addition, SLE sera stimulated the expression of $-180 /$ protein complex only in TCR-positive and not in TCR-negative Jurkat T cells (Figure 13). Interestingly, none of the other antibodies used had a similar effect, and, more importantly, the presence of anti-CD28 did not enhance the effect of anti-CD3 antibody (data not shown). Therefore, anti-TCR antibodies present in the sera of SLE patients can, by activating CaMKIV and CREM, lead to decreased IL-2 production. Our studies do not exclude the possibility that additional factors, including IFN- $\alpha$, can contribute to the increased expression of CREM and the suppression of IL-2 production. Yet, treatment of normal $T$ cells with IFN- $\alpha$ failed to induce the expression of mRNA CREM (unpublished observations, V. Kyttaris, Y.-T. Juang, and G.C. Tsokos).

In summary, this study demonstrates that SLE serum factors enhance the levels of -180 /protein complex in a CaMKIV-dependent manner. The presence of increased amounts of CaMKIV in the nucleus of SLE T cells isolated from the peripheral blood of patients may represent a chronic stimulatory effect of various serum components on the cells. CREM is upregulated in normal $\mathrm{T}$ cells stimulated in vitro, and it has been postulated that this upregulation occurs in time to terminate the IL-2 production so that the immune system is not exhausted (33). In SLE patients, the continuous effect of serum factors leads to an inappropriate decrease of IL-2 production with significant effect on the development of cytotoxic responses $(34,35)$ and the elimination of autoreactive $T$ cells $(4)$, 2 processes that require the presence of IL-2. In addition, defective IL-2 production may curtail the expansion of regulatory $\mathrm{T}$ cells (36). It is interesting to note that the pathway described herein, SLE sera $\rightarrow$ CaMKIV $\rightarrow$ CREM, will affect only genes controlled by the CRE cis element, not other genes. A number of genes, such as CD40 ligand $(37,38)$ and IL-6 $(32)$, are upregulated in SLE and are involved in providing help to $\mathrm{B}$ cells to produce autoantibody. Therefore, although the increased expression of CREM in SLE $T$ cells may represent the effect of anti-TCR/CD3 antibodies present in SLE sera, other $T$ cell defects may be independent of the effect of serum factors (17). Because SLE T cells provide help to B cells to produce most autoantibodies, it is possible that the production of anti-TCR/CD3 antibodies was instigated by $\mathrm{T}$ cells 
overexpressing CD40 ligand. Future studies will delineate the order of appearance of SLE T cell abnormalities and define their importance in the expression of the disease.

\section{Methods}

Patients and controls. Thirty-nine patients fulfilling at least 4 of 11 revised criteria of the American College of Rheumatology for the classification of SLE were studied. All patients were women 21-82 years old and had SLE disease-activity index (SLEDAI) scores ranging from 0 to 12. Five patients with the diagnosis of RA were also included in the study. Medications were discontinued at least 24 hours before venipuncture. Thirty-three normal women served as controls in this study. The protocol was approved by Institutional Review Boards of the Walter Reed Army Institute of Research, the Washington Hospital Center, and the Uniformed Services University of the Health Sciences.

T cells. Primary T cells were purified from human blood within 2-16 hours after venipuncture using the RosetteSep T cell purification kit (StemCell Technologies). More than 95\% of the purified cells were CD3 positive as determined by flow cytometry. Sera were collected and frozen at $-70^{\circ} \mathrm{C}$ until used. Sera were heat-inactivated at $56^{\circ} \mathrm{C}$ for $30 \mathrm{~min}-$ utes and centrifuged before use. Jurkat $\mathrm{T}$ cells were maintained in the laboratory. The Jurkat $\mathrm{T}$ cell subline J.EMS.T3.3 was provided kindly by A. Weiss (University of San Francisco, San Francisco, California, USA). This line does not express TCR/CD3 but expresses all other molecules, including CD28 (39).

Antibodies, plasmids, and reagents. To detect CREM in a specific manner, we generated an anti-human CREM antibody by immunizing rabbits with a peptide with its sequence specific to the $\mathrm{N}$-terminal of human CREM (amino acids 21-34, SKSAHVQTQTGQNS), where the least homology was found among CREM, CREB, and ATF-1. The produced anti-CREM antibody recognized in both human primary $\mathrm{T}$ and Jurkat cells a band migrating to a position level with $26 \mathrm{kDa}$, corresponding to the calculated molecular mass of human CREM $\alpha$. This antibody does not cross-react with CREB or ATF-1, as indicated by its failure to pick up bands of 33 $\mathrm{kDa}$ (ATF-1) or $43 \mathrm{kDa}$ (CREB). Both CREB and ATF-1 antibodies were purchased from Upstate Cell Signaling Solution; normal murine IgG, antiCD4, and anti-c-Jun antibodies were purchased from Santa Cruz Biotechnology Inc.; antibodies against CD28 and CD45 were purchased from BD Biosciences - Pharmingen; anti-CD3 antibody was purchased from Ortho Biotech Products LP; antibodies against CaMKII and CaMKIV were purchased from Transduction Laboratories. The generation and characterization of anti-hnRNP antibody have been described (40).

The expression plasmids of CaMKII(K42M), wild-type CaMKIV, and CaMKIV(K75E) were kindly provided by A.R. Means (Duke University, Durham, North Carolina, USA) and have been described (41, 42). Mutation of K42M in CaMKII and K75E in CaMKIV leads to the expression of inactive enzymes, which are referred to as inactive constructs in this article. Overexpression of these mutated constructs exerts a dominant negative effect because they block the function of endogenous CaMKII or CaMKIV $(43,44)$. The luciferase reporter driven by 2 copies of the -180 element $(-164$ to -189$)$ or the proximal region $(-575$ to +57$)$ of the IL-2 promoter has been described before (5).

Transfection of primary $T$ cells. The transfection of primary $\mathrm{T}$ cells was conducted with the Nucleofector reagent according to the manufacturer's protocols (Amaxa Biosystems) as described before (33). Cell viability remained over $90 \%$ after 24 hours of transfection. Cells were always cotransfected with a $\beta$-gal construct, and its expression was used to normalize for transfection efficiency.

Western blotting. The cellular proteins were separated in SDS gel according to the manufacturer's protocols (Invitrogen Corp.). The proteins were then transferred onto PVDF and blotted with various antibodies as indicated.

CREM and actin RT-PCR. The CREM and actin mRNA levels were determined using RT-PCR as described previously $(5,6)$.

Immunofluorescent staining. Human primary T cells $\left(0.5 \times 10^{6}\right)$ were seeded onto a microscope cover slide precoated with poly-L-lysine (Sigma-Aldrich) in a 24-well plate in RPMI 1640 medium supplemented with $2 \%$ BSA. After 30 minutes of incubation, cells were washed once with PBS and fixed with $4 \%$ paraformaldehyde for 20 minutes. Cells were permeabilized with $0.1 \%$ Triton X-100 in PBS for 5 minutes followed by blocking for 30 minutes with PBS containing $2 \%$ BSA. Cells were stained with anti-CaMKIV (1:100) antibody for 1 hour at room temperature. After washing 3 times with PBS, the cells were stained with Cyanin 3-tagged secondary antibody (Jackson ImmunoResearch Laboratories Inc.). The stained cover glasses were mounted on a glass slide with $10 \mu \mathrm{l}$ of SlowFade Light Antifade with DAPI (Molecular Probes) and sealed with nail polish. The stained samples were inspected by laser scanning confocal fluorescent microscopy using the Radiance 2100 laser scanning system (Bio-Rad Laboratories). All images were collected using a $\times 100$ objective under the same condition.

IL-2 ELISA. IL-2 concentrations were determined as described previously $(5,6)$.

Electrophoresis mobility shift assay. The purification of nuclear proteins and the conduction of electrophoresis mobility shift assay (EMSA) have been described previously $(5,6)$. Notably, polydG.dC, instead of polydI.dC, was used as carrier in all the reactions shown in this study $(5,6)$. The sequences of oligonucleotides used for $I L-2$ promoter -180 site binding have been described (5); the sequence of the M1 oligonucleotide, which was used as cold competitor, represents the wild-type -180 oligonucleotide with 2 nucleotides changed (45). The sequence of the CREM peptide amino acids 21-34 is SKSAHVQTQTGQNS. The sequence of the control peptide hnRNP D0 amino acids 263-275 is YQQQQQWGSRGGF.

Sera fractionation. SLE or normal sera were fractionated by ProteoExtract IgG Removal Kit (Calbiochem). Briefly, sera were diluted 1:10 into binding buffer and then loaded onto the column that retains IgG. After collection of the flow-through, the column was thoroughly washed with the same binding buffer before the bound IgG was eluted by $10 \mathrm{mM}$ glycine. The eluate was immediately mixed with $10 \mathrm{mM}$ Tris, $\mathrm{pH}$ 8.0, to neutralize the $\mathrm{pH}$. The purity of each fraction was assayed by SDS gel electrophoresis followed by staining. The functional integrity of the IgG that was purified was implied by the fact that similarly purified anti-CREM antibody maintained its function in Western blots.

Statistical analysis. Data are reported as mean \pm SEM. SPSS version 11.5.1 (SPSS Inc.) was used for statistical analysis.

\section{Acknowledgments}

This work was supported by NIH grants RO1 AI42269 and RO1 AI49954. We thank A. Weiss, T. Brumeanu, R. Wange, D. Farber, and R. Peckham for helpful reagents and discussions, and M. Zidanic for expert help with confocal microscopy. We also thank A. Weinstein, R. Bagati, P. De Marco, B. Wallit, S. Timbil, and V. Logan for their help with the recruitment of the research subjects. The opinions expressed herein are those of the authors and do not represent those of the Department of Defense.

Received for publication July 28, 2004, and accepted in revised form January 5, 2005.

Address correspondence to: George C. Tsokos, Walter Reed Army Institute of Research, Building 503, Room 1A32, Silver Spring, Maryland 20910, USA. Phone: (301) 319-9911; Fax: (301) 3199133; E-mail: gtsokos@usuhs.mil. 
1. Linker-Israeli, M., et al. 1983. Defective production of interleukin 1 and interleukin 2 in patients with systemic lupus erythematosus (SLE). J. Immunol. 130:2651-2655.

2. Theofilopoulos, A.N., and Dixon, F.J. 1985. Murine models of systemic lupus erythematosus. $A d v$. Immunol. 37:269-390.

3. Iliopoulos, A.G., and Tsokos, G.C. 1996. Immunopathogenesis and spectrum of infections in systemic lupus erythematosus. Semin. Arthritis Rheum. 25:318-336.

4. Kovacs, B., Vassilopoulos, D., Vogelgesang, S.A., and Tsokos, G.C. 1996. Defective CD3-mediated cell death in activated $\mathrm{T}$ cells from patients with systemic lupus erythematosus: role of decreased intracellular TNF-alpha. Clin. Immunol. Immunopathol. 81:293-302.

5. Solomou, E.E., Juang, Y.T., Gourley, M.F., Kammer, G.M., and Tsokos, G.C. 2001. Molecular basis of deficient IL-2 production in T cells from patients with systemic lupus erythematosus. J. Immunol. 166:4216-4222.

6. Tenbrock, K., Juang, Y.-T., Gourley, M.F., Nambiar, M.P., and Tsokos, G.C. 2002. Antisense cyclic adenosine $5^{\prime}$-monophosphate response element modulator up-regulates IL-2 in T cells from patients with systemic lupus erythematosus. J. Immunol. 169:4147-4152.

7. De Cesare, D., Fimia, G.M., and Sassone-Corsi, P. 1999. Signaling routes to CREM and CREB: plasticity in transcriptional activation. Trends Biochem. Sci. 24:281-285.

8. Lee, K.A., and Masson, N. 1993. Transcriptional regulation by CREB and its relatives. Biochim. Biophys. Acta. 1174:221-233.

9. Powell, J.D., Lerner, C.G., Ewoldt, G.R., and Schwartz, R.H. 1999. The -180 site of the IL-2 promoter is the target of CREB/CREM binding in $\mathrm{T}$ cell anergy. J. Immunol. 163:6631-6639.

10. Tada, Y., Nagasawa, K., Yamauchi, Y., Tsukamoto, H., and Niho, Y. 1991. A defect in the protein kinase $\mathrm{C}$ system in $\mathrm{T}$ cells from patients with systemic lupus erythematosus. Clin. Immunol. Immunopathol. 60:220-231.

11. Cedeno, S., et al. 2003. Defective activity of ERK-1 and ERK-2 mitogen-activated protein kinases in peripheral blood $\mathrm{T}$ lymphocytes from patients with systemic lupus erythematosus: potential role of altered coupling of Ras guanine nucleotide exchange factor hSos to adapter protein Grb2 in lupus T cells. Clin. Immunol. 106:41-49.

12. Kammer, G.M. 2002. Deficient protein kinase A in systemic lupus erythematosus: a disorder of T lymphocyte signal transduction. Ann. N. Y. Acad. Sci. 968:96-105.

13. Vassilopoulos, D., Kovacs, B., and Tsokos, G.C. 1995. TCR/CD3 complex-mediated signal transduction pathway in $T$ cells and cell lines from patients with systemic lupus erythematosus. J. Immunol. 155:2269-2281.

14. de Groot, R.P., Ballou, L.M., and Sassone-Corsi, P. 1995. Positive regulation of the nuclear activator CREM by the mitogen-induced p70 S6 kinase. Immunobiology. 193:155-160.

15. Krebs, J., Wilson, A., and Kisielow, P. 1997. Calmodulin-dependent protein kinase IV during T-cell development. Biochem. Biophys. Res. Commun. 241:383-389.

16. Blanco, P., Palucka, A.K., Gill, M., Pascual, V., and
Banchereau, J. 2001. Induction of dendritic cell differentiation by IFN-alpha in systemic lupus erythematosus. Science. 294:1540-1543.

17. Winfield, J.B., and Mimura, T. 1992. Pathogenetic significance of anti-lymphocyte autoantibodies in systemic lupus erythematosus. Clin. Immunol. Immunopathol. 63:13-16.

18. Dash, P.K., Karl, K.A., Colicos, M.A., Prywes, R., and Kandel, E.R. 1991. cAMP response element-binding protein is activated by $\mathrm{Ca} 2+/$ calmodulin- as well as cAMP-dependent protein kinase. Proc. Natl. Acad. Sci. U. S. A. 88:5061-5065.

19. Bito, H., Deisseroth, K., and Tsien, R.W. 1996. CREB phosphorylation and dephosphorylation: a $\mathrm{Ca}(2+)$ - and stimulus duration-dependent switch for hippocampal gene expression. Cell. 87:1203-1214

20. Groussin, L., Massias, J.F., Bertagna, X., and Bertherat, J. 2000. Loss of expression of the ubiquitous transcription factor cAMP response element-binding protein (CREB) and compensatory overexpression of the activator CREMtau in the human adrenocortical cancer cell line H295R. J. Clin. Endocrinol. Metab. 85:345-354.

21. Hummler, E., et al. 1994. Targeted mutation of the CREB gene: compensation within the CREB/ATF family of transcription factors. Proc. Natl. Acad. Sci. U. S. A. 91:5647-5651

22. de Groot, R.P., Ballou, L.M., and Sassone-Corsi, P. 1994. Positive regulation of the cAMP-responsive activator CREM by the p70 S6 kinase: an alternative route to mitogen-induced gene expression. Cell. 79:81-91.

23. Jensen, K.F., Ohmstede, C.A., Fisher, R.S., and Sahyoun, N. 1991. Nuclear and axonal localization of $\mathrm{Ca} 2+$ /calmodulin-dependent protein kinase type $\mathrm{Gr}$ in rat cerebellar cortex. Proc. Natl. Acad. Sci. U. S. A. 88:2850-2853.

24. Hanissian, S.H., Frangakis, M., Bland, M.M., Jawahar, S., and Chatila, T.A. 1993. Expression of a $\mathrm{Ca} 2+/$ calmodulin-dependent protein kinase, $\mathrm{CaM}$ kinase-Gr, in human T lymphocytes. Regulation of kinase activity by $\mathrm{T}$ cell receptor signaling. J. Biol. Chem. 268:20055-20063.

25. Frangakis, M.V., Chatila, T., Wood, E.R., and Sahyoun, N. 1991. Expression of a neuronal Ca2+/ calmodulin-dependent protein kinase, CaM kinase$\mathrm{Gr}$, in rat thymus. J. Biol. Chem. 266:17592-17596.

26. Wayman, G.A., Tokumitsu, H., and Soderling, T.R. 1997. Inhibitory cross-talk by cAMP kinase on the calmodulin-dependent protein kinase cascade. J. Biol. Chem. 272:16073-16076.

27. MacNicol, M., and Schulman, H. 1992. Cross-talk between protein kinase $\mathrm{C}$ and multifunctional $\mathrm{Ca} 2+/$ calmodulin-dependent protein kinase. J. Biol. Chem. 267:12197-12201.

28. Sun, P., Lou, L., and Maurer, R.A. 1996. Regulation of activating transcription factor- 1 and the cAMP response element-binding protein by $\mathrm{Ca} 2+$ / calmodulin-dependent protein kinases type I, II, and IV. J. Biol. Chem. 271:3066-3073.

29. Heist, E.K., Srinivasan, M., and Schulman, H. 1998. Phosphorylation at the nuclear localization signal of $\mathrm{Ca} 2+/$ calmodulin-dependent protein kinase II blocks its nuclear targeting. J. Biol. Chem. 273:19763-19771.

30. Anderson, K.A., and Kane, C.D. 1998. Ca2+/ calmodulin-dependent protein kinase IV and calcium signaling. Biometals. 11:331-343.
31. Selbert, M.A., et al. 1995. Phosphorylation and activation of $\mathrm{Ca}(2+)$-calmodulin-dependent protein kinase IV by $\mathrm{Ca}(2+)$-calmodulin-dependent protein kinase Ia kinase. Phosphorylation of threonine 196 is essential for activation. J. Biol. Chem. 270:17616-17621.

32. Linker-Israeli, M., et al. 1999. Association of IL-6 gene alleles with systemic lupus erythematosus (SLE) and with elevated IL-6 expression. Genes Immun. 1:45-52.

33. Tenbrock, K., Juang, Y.-T., Tolnay, M., and Tsokos, G.C. 2003. The cyclic adenosine 5'-monophosphate response element modulator suppresses IL-2 production in stimulated $T$ cells by a chromatin-dependent mechanism. J. Immunol. 170:2971-2976.

34. Tsokos, G.C. 1999. Overview of cellular immune function in systemic lupus erythematosus. In Systemic lupus erythematosus. R.G. Lahita, editor. Academic Press. New York, New York, USA. 17-54.

35. Stohl, W. 1999. Impaired non-antigen specific T cell cytolytic activity in SLE. In Lupus: molecular and cellular pathogenesis. G.M. Kammer and G.C. Tsokos, editors. Humana Press Inc. Totowa, New Jersey, USA. 299-311.

36. Malek, T.R., and Bayer, A.L. 2004. Tolerance, not immunity, crucially depends on IL-2. Nat. Rev. Immunol. 4:665-674.

37. Koshy, M., Berger, D., and Crow, M.K. 1996. Increased expression of CD40 ligand on systemic lupus erythematosus lymphocytes. J. Clin. Invest. 98:826-837.

38. Desai-Mehta, A., Lu, L., Ramsey-Goldman, R., and Datta, S.K. 1996. Hyperexpression of CD40 ligand by $\mathrm{B}$ and $\mathrm{T}$ cells in human lupus and its role in pathogenic autoantibody production. J. Clin. Invest. 97:2063-2073.

39. Weiss, A., and Stobo, J.D. 1984. Requirement for the coexpression of $\mathrm{T} 3$ and the $\mathrm{T}$ cell antigen receptor on a malignant human T cell line. J. Exp. Med. 160:1284-1299.

40. Tolnay, M., Vereshchagina, L.A., and Tsokos, G.C. 1999. Heterogeneous nuclear ribonucleoprotein $\mathrm{D} 0 \mathrm{~B}$ is a sequence-specific DNA-binding protein. Biochem. J. 338:417-425.

41. Chatila, T., Anderson, K.A., Ho, N., and Means, A.R. 1996. A unique phosphorylation-dependent mechanism for the activation of $\mathrm{Ca} 2+/$ calmodulindependent protein kinase type IV/GR. J. Biol. Chem. 271:21542-21548.

42. Cruzalegui, F.H., and Means, A.R. 1993. Biochemical characterization of the multifunctional $\mathrm{Ca} 2+/$ calmodulin-dependent protein kinase type IV expressed in insect cells. J. Biol. Chem. 268:26171-26178.

43. Enslen, H., Tokumitsu, H., Stork, P.J., Davis, R.J., and Soderling, T.R. 1996. Regulation of mitogenactivated protein kinases by a calcium/calmodulindependent protein kinase cascade. Proc. Natl. Acad. Sci. U. S. A. 93:10803-10808.

44. Nielson, C.P., and Wingett, D. 2002. Endothelial cell and cAMP regulation of T-cell CD40 ligand: relevance of calcium/calmodulin-dependent kinase IV signalling. Immunology. 105:430-440.

45. Solomou, E.E., Juang, Y.T., and Tsokos, G.C. 2001. Protein kinase C-theta participates in the activation of cyclic AMP-responsive element-binding protein and its subsequent binding to the -180 site of the IL-2 promoter in normal human T lymphocytes. J. Immunol. 166:5665-5674. 